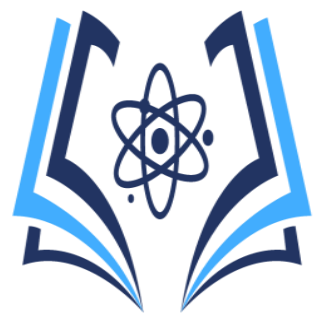

\title{
La COVID-19 un reto para la ciencia mundial
}

\author{
COVID-19 a challenge for world science \\ COVID-19 un reto
}

\author{
Cristian Paul Vera Zambrano ${ }^{(1)}$ \\ Karla Valeria Bello Mendoza ${ }^{(2)}$ \\ Luis Marcelo Narváez Bonoso ${ }^{(3)}$ \\ Ana Lucia San Andrés Mendoza ${ }^{(4)}$ \\ ${ }^{1}$ Hospital de Especialidades Portoviejo cristian.cv742@gmail.com \\ ${ }^{2}$ Universidad Técnica de Manabí valeriabello98@hotmail.com \\ ${ }^{3}$ Universidad Técnica de Manabí, marcelovn06@gmail.com \\ ${ }^{4}$ Universidad Técnica de Manabí, anitasanandres2002@gmail.com
}

Contacto: cristian.cv742@gmail.com

Recibido: 20-07-2021

Aprobado: 02-08-2021

\section{Resumen}

El presente artículo ofrece una reflexión académica que, en término; panorámicos, busca presentar la serie de retos que plantea la crisis sanitaria del COVID-19 para poder avanzar hacia un desarrollo social basado en la sustentabilidad; El nuevo coronavirus (2019-nCoV, SARS-CoV-2 o COVID19) se volvió un reto para todo el mundo, hasta el momento ha afectado a más de 180 países de casi todos los continentes, fue declarado por la Organización Mundial de la Salud como pandemia por su alcance mundial y hasta la fecha, ha sobrepasado los dos millones de casos de infectados. El número exponencialmente creciente de casos en el mundo, refleja en parte la rápida transmisión del COVID-19, que se traduce en una dura prueba para los sistemas de salud de los países más afectados. Materiales y métodos se realizó un estudio observacional, descriptivo y bibliométrico en los artículos sobre COVID-19 un reto para ciencia, publicados en el año 2020 hasta la fecha. Resultados contar con datos detallados y precisos permitirá comprender y hacer un seguimiento del alcance de esta pandemia y fortalecer los esfuerzos de prevención y respuesta, ya que es un fenómeno sin precedentes en el último siglo en relación al número de personas y países que ha afectado y al impacto en las dinámicas sociales y económicas del mundo actual.

Palabras clave: pandemia, retos, SARS-CoV-2, transmisión de enfermedad infecciosa

\section{Abstract}

This article offers an academic reflection that, in panoramic terms, seeks to present the series of challenges posed by the health crisis of COVID-19 in order to advance towards social development based on sustainability; The new coronavirus (2019-nCoV, SARS-CoV-2 or COVID-19) has become a challenge 
for the whole world, so far it has affected more than 180 countries on almost all continents, it was declared by the World Organization of Health as a pandemic due to its global scope and to date, it has exceeded two million cases of infected. The exponentially growing number of cases in the world reflects in part the rapid transmission of COVID-19, which translates into a severe test for the health systems of the most affected countries. Materials and methods an observational, descriptive and bibliometric study was carried out in the articles on COVID-19 a challenge for science, published in 2020 to date. Results Having detailed and accurate data will allow us to understand and monitor the scope of this pandemic and strengthen prevention and response efforts, since it is a phenomenon without precedent in the last century in relation to the number of people and countries that it has affected and to the impact on the social and economic dynamics of the world today.

Keywords: Pandemic, challenges, SARS-CoV-2, infectious disease transmission

\section{Introducción}

Hace algunos años se hablaba de una tercera guerra mundial y los impensables estragos que esta haría en la humanidad, pero pocos imaginaron que hoy estuviésemos en guerra contra un enemigo común que la ciencia todavía no ha podido controlar, pues aún no cuenta con todas las armas necesarias. Los científicos, médicos, enfermeros, otros trabajadores del sistema de salud y de múltiples áreas del conocimiento se encuentran de frente al combate, ya sea desde un laboratorio o en el diseño de un modelo de predicción científica, o directamente con el paciente. Somos el ejército de batas blancas creado por esta Revolución para combatir, sin miedo, bajo el concepto de salvar no solo una vida, sino a la humanidad. Nada supera ese actuar, nada supera esa consagración y a esos valores.
Cuando por primera vez se reportó en enero de 2020 el brote de una enfermedad respiratoria grave en Wuhan, China, el mundo reaccionó con cierta indiferencia; cuando se describió que la enfermedad llamada COVID-19 era causada por un virus denominado SARS-CoV-2, su dispersión ya era incontenible. Se prendieron las alarmas, sin embargo, el brote rápidamente se convirtió en una pandemia reconocida por la Organización Mundial de la Salud (OMS). Al 29 de noviembre, se habían notificado a dicha organización, 61869330 casos confirmados de la COVID-19 y 1448896 muertes. En las Américas desde el ingreso del SARS-CoV-2, el 20 de enero, se habían reportado 26216515 casos, mientras en el Perú -cuyo primer caso confirmado se reportó el 6 de marzo- se han registrado 958324 casos confirmados y 35839 fallecidos. (1)

La emergencia de esta enfermedad, se ha explicado por el surgimiento de un nuevo coronavirus humano con incrementados factores de virulencia. El número exponencialmente creciente de casos en el mundo, refleja en parte la rápida transmisión del COVID-19, alcanzando la etapa de propagación en la comunidad, dada la susceptibilidad del $100 \%$ de la población mundial. Contar con datos detallados y precisos permitirá comprender y hacer un seguimiento del alcance de esta pandemia y fortalecer los esfuerzos de prevención y respuesta, en virtud que hasta el momento los tratamientos son experimentales y la vacuna podría estar disponible a mediano o largo plazo.

América Latina y el Caribe es la región con más desigualdades en el mundo y también la más urbanizada entre las regiones en desarrollo, lo que expone a un gran porcentaje de su población al contagio en condiciones de desprotección. De otro lado esta pandemia apareció súbitamente en un escenario económico, social y político complejo, en tiempos de bajo crecimiento económico y elevados niveles de informalidad laboral. Este escenario 
convirtió a nuestra región en el epicentro de la pandemia COVID-19, y ha generado un contexto propicio que podría conllevar a una crisis alimentaria, humanitaria y política, si no afrontamos el problema de manera integral.

El enfrentamiento al COVID-19 impone importantes retos a los profesionales de la salud en el mundo relacionados, fundamentalmente, con los cuidados de la salud de la población y con la prevención y control de infecciones en la comunidad y en las instituciones de salud. La complejidad de la atención de pacientes con enfermedades infecciosas que generan una proporción significativa de casos que requieren cuidados críticos y ventilación asistida, orientan la necesidad de fortalecer la formación profesional de los que participan en los cuidados de urgencia o emergencia en los diferentes niveles del sistema de salud (2)

Las medidas implementadas para el control de la epidemia en la nación son similares a las utilizadas en el control del SARS y el COVID-19 en China y están diseñadas con objetividad para identificar su impacto en la prevención y control de este problema de salud. Para los programas de control de infecciones en instituciones de salud, la infección por COVID-19 constituye un reto de mayor importancia, pues se requieren sólidos conocimientos y habilidades sobre prevención de la transmisión de infecciones en estos centros y la disponibilidad de medios de protección individual, siempre limitados por nuestras posibilidades financieras. No obstante, el desafío mayor hoy lo constituye la capacitación de los trabajadores de la salud en estos temas y el logro de la prevención de la transmisión de infecciones en los centros de salud.

La humanidad sufre hoy una situación grave: casi 2 millones de personas se han afectado por una angustiosa enfermedad, la COVID-19, provocada por un virus poco conocido, el SARS-CoV-2, que ha provocado alrededor de 126604 de fallecidos,
Vol. 5, Nro. 2, Publicado: 2021-12-31

incluidos jóvenes, aunque la afectación es mayor en personas de la tercera edad y aquellas que tienen factores de riesgo que los hacen más vulnerables como la hipertensión arterial, la diabetes mellitus, entre otras. La Organización Mundial de la Salud hace referencia a que el SARS-CoV-2 no va a desaparecer, lo cual implica que la humanidad tendrá que prepararse para enfrentar prospectivamente esta realidad, que complica más la situación epidemiológica de los diferentes países. (3)

La COVID-19 ha puesto también al descubierto nuestras serias limitaciones en el conocimiento científico sobre los mecanismos de transmisión del SARS-CoV-2, su fisiopatología y respuesta inmune humoral y celular, la presentación clínica, la disponibilidad de diagnósticos de laboratorio, tratamiento farmacológico y no farmacológico, y sobre todo de las estrategias de control que sean eficaces. Muchos de estos conocimientos están en pleno proceso de generación por su inexorable necesidad.

Durante el curso de la pandemia se ha actuado con las pocas evidencias científicas disponibles, lo cual en la mayoría de países no ha permitido tener mucho éxito para la prevención y control de la infección. Hubo y sigue habiendo serias controversias entre los médicos sobre la utilidad y limitaciones de las diferentes intervenciones. Muchas de estas controversias son alimentadas por los medios de comunicación y las redes sociales. También influyen el contexto político de los países y los comercializadores de diferentes productos e insumos médicos, cada uno con intereses particulares, pero que en conjunto limitan una acción concertada contra el avance de la pandemia.

En la actualidad son las políticas públicas las que deben trabajar de la mano con los procesos y la evolución científica, ya que son ellos que se deben comprometer tanto como los investigadores, ya que son ellos los encargados y llamados a tratar de proteger y salvaguardar a la población, sin que nadie 
quede atrás, es decir, que en esta nueva era que estamos viviendo la ciencia debe tener un espacio de gran relevancia en el mundo. A través de esta gran crisis los postulados científicos se han vuelto más irrefutables que nunca, ya que al momento de que se va desarrollando la comunidad científica, va existiendo más seguridad y menos riesgos.; no olvidemos que, para poder lograr estos objetivos de salvaguardar y proteger a la población, nos bajamos en la cooperación internacional en un enfoque de derechos humanos.

En unos pocos meses el medio se vio en una gran vuelta de adversidad que pasará a la historia como una de las grandes pandemias que nosotros hemos sufrido en los últimos años. Es ahí donde la pérdida de tantas vidas dejará una huella en todos nosotros y las repercusiones psicológicas de la incertidumbre es otro elemento impactante que conducirá a cambios en nuestro futuro a medida que esta pandemia se termine. Los científicos, médicos, enfermeros, otros trabajadores del sistema de la salud y de múltiples áreas del conocimiento se encuentran de porte a la batalla, ya sea desde una botica o en el diseño de un arquetipo de augurio científica. 'la ciencia fue, es y será siempre un derecho humano fundamental. Igual que la salud, la educación, la vivienda". (4)

La mayor afectación ha sido a personas de la tercera edad y aquellas que tienen factores de riesgo que los llegan hacer más vulnerables, tales como la hipertensión arterial, la diabetes mellitus, entre otros más. La Organización Mundial de la Salud hace una gran referencia a que el SARS-CoV-2 no va a desaparecer, es decir esto implicaría que toda la humanidad tendrá que prepararse para poder enfrentar esta realidad, la cual complicará más la situación epidemiológica de la mayoría de los países.

Entre los muchos daños directos y colaterales que está produciendo la COVID-19, uno de los apartes mencionado seguramente sea como nos impide
Vol. 5, Nro. 2, Publicado: 2021-12-31

considerar con perspectiva. Es aceptablemente razonable, pues diario nos vemos expuestos a una masa de mensajes, dígitos, rumores y opiniones que alimentan la intranquilidad y la incertidumbre que sentimos cerca de la bacteria y las consecuencias, ya sean sanitarias o económicas, que se puedan acontecer para nosotros y nuestros seres queridos. Aunque los mecanismos fisiopatológicos no están totalmente esclarecidos, al ser la COVID-19 una gran enfermedad, muchos de estos estudios científicos nos sugieren que en nuestro sistema inmunológico podría no defendernos de este enemigo mortal y, que en consecuencia a esto fallan los mecanismos de control y de respuestas fisiológica.

El científico de datos Youyang $\mathrm{Gu}$, se considera realista, por lo que cuando vio las proyecciones diversas acerca del COVID-19 como un modelo en el cual se esperaban aproximadamente dos millones de muertes en Estados Unidos para el verano y 60.000 para el siguiente, empezó a dudar de la calidad que poseía los modelos informáticos por lo que tomo la decisión de crear uno propio. (6) El objetivo de la investigación que el realizaba era poder obtener un modelo más preciso, el investigador no tenía ningún tipo de experiencia referente a enfermedades infecciosas, pero si poseía experiencia en modelos de datos estadísticos por lo que se volvió más preciso.

En tal sentido, la comunidad médica y científica mundial lleva a cabo ingentes esfuerzos en la investigación biomédica, clínica y epidemiológica, abarcadoras de las dimensiones biológica, psicológica, social y cultural antropológica del hombre sano, enfermo o en riesgo de enfermar, la familia, la comunidad y el ambiente, ante la situación problemática ocurrida, traducida en un problema de salud, en cuyo cuerpo teórico referencial existen brechas importantes, por lo que las acciones de atención médica integral se complementan obligatoriamente con la investigación científica para poder dar solución al problema de salud que a la vez 
es un problema científico y complejo que conlleva un abordaje inter-transdisciplinario, en las esferas de actuación primaria, secundaria y terciaria y con participación colectiva de los actores.

El modelo que presento $\mathrm{Gu}$, está basado en la combinación del aprendizaje automático con un simulador clásico de enfermedades infecciosas llamado modelo SEIR, la cual busca incluir a las personas que son más susceptibles, expuestas, contagiosas, recuperadas y fallecidas. Es por ello que desde la UNESCO (Organización de Naciones Unidas para la Educación, la Ciencia y la Cultura) se impulsa a la población a defenderse de todos los actores políticos, civiles y académicos que quieran impedir la participación en conjunto con la ciencia.

Gracias a este auténtico avance de la tecnología, vamos a poder pasar un arrebato sanitario que este no existía hace algunas décadas atrás, hubiera arrojado una cifra de víctimas mucho mayor, y esto se va hacer además de un periodo de tiempo mucho más corto tenemos en cuenta que si aprendemos el valor de la innovación para así poder luchar con este tipo de situaciones, y cada uno sigue apostando como sociedad para la ciencia y la tecnología, tomando conciencia conseguiremos poder revenir o por lo menos disminuir muy notablemente los efectos de nuevas pandemias (8)

\section{Materiales y Métodos}

Se realizó un estudio observacional, descriptivo y bibliométrico en los artículos sobre COVID-19 un reto para ciencia, publicados en revistas médicas entre el 1ro de enero de 2020 y el 10 de junio de 2021. Se seleccionaron aquellas revistas pertenecientes a las universidades de ciencias médicas de diferentes países $(n=50)$. Para la recolección de la información se accedió al portal electrónico de las revistas desde el sitio web de Infomed, Scopus, Eric. El día 02 de enero de 2021 se descargó cada artículo en sus formatos (PDF/HTML/XML) e idiomas disponibles (español/inglés).

\section{Resultados}

La investigación realizada bajo la revisión de muchos artículos científicos de diferente revista de divulgación, se encontraron muchos cuestionamientos de cómo enfrentar las aéreas afectas a raíz de la aparición del Covid-19. En el caso de la ciencia pura, el principal objetivo es el descubrimiento de la verdad científica, mientras que en la ciencia aplicada es la obtención de un resultado que pueda ser incorporado a la práctica social. De 50 revisiones en revistas consultadas, 32 presentaron al menos un artículo sobre la COVID-19 y 18 eran temas afines a sus complicaciones.

Sin embargo, los retos a considerar son múltiples:

1) Mantener en cuarentena prolongada a una población mayoritariamente pobre que depende del trabajo diario para subsistir va a resultar difícil, aun sabiendo que sin cuarentena ni aislamiento social las tasas de transmisión serán muy altas y la duración de la pandemia podría prolongarse en el tiempo;

2) Anticipar la llegada de un nuevo brote, asociado a otros factores como: influenza estacional y dengue que podría complicar el panorama epidemiológico de la región;

3) Prever los efectos sobre las tasas de contagio a nivel Nacional o Regional;

4) Enfrentar un problema de salud pública de tal magnitud en un escenario de limitados recursos económicos, podría comprometer la aplicación de adecuadas estrategias de prevención, la necesaria realización a gran escala de tests de diagnóstico rápido, lo cual se considera de vital importancia para rastrear el virus, comprender la epidemiología local y suprimir la transmisión; y, el adecuado cumplimiento de protocolos de tratamiento ambulatorio e intrahospitalario; 
Periodo. Julio - Diciembre 2021

Vol. 5, Nro. 2, Publicado: 2021-12-31

5) Asumir las consecuencias a mediano y largo plazo de las medidas antes mencionadas sobre economías vulnerables y en muchos casos ya comprometidas.

\section{Discusión}

El presente trabajo ayuda a comprender los efectos y los desafíos del Covid-19, los principales desafíos tienen que ver con la imprevisibilidad de la enfermedad a nivel mundial. Ya tenemos claro el impacto que ha tenido la presencia de esta pandemia. Por el momento, la tasa de mortalidad es muy elevada para aquellos que tienen 70 o más años de edad, así como personas con afecciones preexistentes. Si bien la población más joven corre menor riego de complicaciones, es indispensable que las campañas de prevención estén dirigidas a toda la población para poder reducir el riesgo de contagio. (9)

El COVID-19 está suponiendo una dura prueba para los sistemas de salud de los países más afectados por la pandemia, seguramente, es todavía más duro para Latinoamérica. Los retos que ha representado esta pandemia, muchos han tenido sus bases en las lecciones aprendidas en sucesos pandémicos anteriores. La evidencia emergente sugiere que las personas infectadas con SARS-CoV-2 podrían estar propagando el virus sin reconocer o antes de reconocer los síntomas. Esto haría que las medidas de control que fueron efectivas contra el SARS-CoV-1 sean menos efectivas contra este sucesor.

Entre los principales desafíos para una distribución global figura la producción de las dosis necesarias para acabar con la epidemia. "Se necesitan varios miles de millones de dosis de vacunas y eso es algo que no se ha visto nunca en la historia", (10). Hasta el momento, a nivel mundial se han logrado producir cientos de millones de vacunas con fármacos desarrollados durante décadas. Ahora, tras pocos meses de investigación, las farmacéuticas deben disponer de miles de toneladas de materiales, personal y se deberá desplegar un gran músculo industrial para fabricarlas.
La pandemia no solo ha desafiado la salud pública, sino que ha puesto en peligro puestos de trabajo en las empresas y sectores productivos claves, especialmente el comercio mayorista y minorista; comunitarios; turismo y gastronomía; actividades inmobiliarias, empresariales y de alquiler, y las manufacturas., perturbando a nivel económico y social los medios de vida de millones de personas, incluyendo su bienestar psicosocial. La tarea es titánica y la meta es lo se conoce como 'inmunidad colectiva', que se logra cuando existen suficientes personas inmunes para neutralizar la propagación de un virus. Se estima que para vencer al SARS-CoV-2 es necesario que al menos el $60 \%$ de la población global sea inmune y para ello se necesitan unos 4.700 millones de dosis.

\section{Conclusiones}

Las principales potencias mundiales y las grandes compañías farmacéuticas han volcado sus esfuerzos para conseguir una o varias vacunas que pongan freno a la pandemia global del Covid-19 y cientos de equipos científicos a nivel internacional han dado pasos prometedores para lograr el cometido. A medida que avanza la carrera contrarreloj por una vacuna contra el nuevo coronavirus, una futura distribución universal de los antídotos enfrenta numerosos obstáculos. El precio, la fabricación y los intereses políticos son algunos desafíos para lograr un suministro general y ecuánime.

Los países latinoamericanos no tienen medios, recursos, tecnología ni la capacidad de acción para seguir el ejemplo de Corea del Sur: emprender una campaña masiva para examinar a la población, independientemente de si tienen síntomas o no y, en un marco de transparencia absoluta, hacer pruebas masivas para detectar el virus. Lo que sí puede hacer la región es apostar por la toma de medidas más drásticas lo antes posible, ceñidas a la evidencia científica y a la experiencia de otros países que han resultado exitosas para la gestión de los brotes. Se 
Periodo. Julio - Diciembre 2021

Vol. 5, Nro. 2, Publicado: 2021-12-31

trata de aprovechar este mayor margen de acción que resulta vital para compensar las debilidades que tiene la región en cuanto a medios humanos y técnicos, así como las insuficiencias financieras.

El reto de la educación en tiempos de la pandemia por Covid-19, más allá de observarla como una catástrofe que nos rebasaría como efecto de una equivocada política pública, que no previó la posibilidad de estos escenarios. Debería concientizarnos sobre el rumbo de la educación futura, el cual ya nos alcanzó. Deberíamos tomar esta oportunidad para reordenar desde los contenidos de los cursos que impartimos hasta nuestras herramientas de aprendizaje. El Estado tiene la obligación y el deber de reconocer el trabajo del docente que, no obstante, la falta de recursos para su capacitación, y quien a través del desarrollo de la Pandemia por el Covid 19, en todo momento estuvo manteniendo la firme convicción de su labor. Esta pandemia sin duda marcará un hito para la reflexión de la salud pública, de su papel y de la clara necesidad de que exista un mayor activismo científico a favor de fortalecer los sistemas de salud pública. Viviremos meses muy amargos, en los que a los graves daños a la salud y la sobrecarga del sistema de salud se agregarán problemas sociales y económicos dolorosos. Al tiempo que atendemos estos temas urgentes, debemos empezar a trabajar en nuestro siguiente reto.

La COVID-19 ha traído a la humanidad importantes enseñanzas: aprender del pasado, necesidad de estar bien informados, valor de la ciencia en cuanto a la toma de decisiones, impacto social de la responsabilidad individual y valor de la solidaridad; además, se ha confirmado que los problemas sanitarios están siempre sujetos a la interacción de cada civilización con su entorno, lo que depende, en esencia, del carácter de la relación hombrenaturaleza. La situación crítica que el mundo enfrenta hoy debe ser vista desde todas las dimensiones; se ha ido estructurando a partir de múltiples problemas ambientales, económicos, políticos y sociales hasta coexistir con una crisis sanitaria, social y económica, lo que connota la urgencia de un accionar integrado y responsable.

\section{Referencias}

1. Geo-Hub COVID-19. Sistema de informacion para la Region de las Americas. [Online].; 2020 [cited 2021 06 13. Available from: https://paho-covid19-response-who.hub.arcgis.com/.

2. Barrientos Gutiérrez T, Alpuche Aranda C, Lazcano Ponce E, Pérez Ferrer C, Rivera Dommarco J. La Salud Publica en la primera ola: una agenda para la cooperacion ante el Covid-19. Licencia CC. 2020;: p. 9.

3. Perez V. Reto para la ciencia mundial. Anales De La Academia De Ciencias De Cuba. 2020;: p. 16.

4. Brito L. el nuevo rol de la ciencia en el mundo. El pais. 2021 abril 26: p. 12.

5. OMS. Brote de enfermedad por coronavirus (COVID-19): orientaciones para el público. Organizacion Mundial de la Salud. 2020;: p. 18.

6. Roberts S. La ciencia es un método para encontrar la verdad, no la verdad en sí. MIT Technology Review. 2021;: p. 17.

7. UNESCO. enfrentando la emergencia en la region. Organización de Naciones Unidas para la Educación, la Ciencia y la Cultura. 2021;: p. 14.

8. Praeli YS. mongabay. [Online].; 2020. Available from: https://es.mongabay.com/2020/04/covid-19-trabajocientifico-latinoamerica/. 
9. Benveniste L. Publicando voces. [Online].; 2020. Available from: https://blogs.worldbank.org/es/voces/desafios-y-respuestas-de-la-covid-19-una-vision-de-america-latina-yel-caribe.

10. Plazas N. France 24. [Online].; 2020 [cited 202106 14. Available from: https://www.france24.com/es/ee-uuy-canad\%C3\%A1/20201015-eeuu-elecciones-mascarilla-politizada-trump-biden-salud-pandemia. 